\title{
Star formation: The role of high resolution radio surveys
}

\author{
R. J. Beswick*, M. K. Argo, T. W. B. Muxlow, N. Wrigley, J. F. Radcliffe \\ Author Jodrell Bank Centre for Astrophysics/e-MERLIN, The University of Manchester, \\ M13 9PL, UK \\ E-mail: Robert.Beswick@manchester.ac.uk
}

\begin{abstract}
The role of star-formation is critical for galaxy evolution. It is a ubiquitous process in galaxies that is both influenced by, and drives their evolution. In this article we will review the current status of high resolution studies of star-formation processes within galaxies from the local to the distant Universe. In particular this review will focus on the state-of-the-art radio surveys that are now underway which provide some of the highest angular resolution and unobscured views of star-formation in galaxies. These surveys significantly add to our understanding of the processes of star-formation and provide the first glimps of what will be achievable with the SKA in the coming decades.
\end{abstract}

EXTRA-RADSUR2015 (*)

20-23 October 2015

Bologna, Italy

(*) This conference has been organized with the support of the Ministry of Foreign Affairs and International Cooperation, Directorate General for the Country Promotion (Bilateral Grant Agreement ZA14GR02 - Mapping the Universe on the Pathway to SKA)

\footnotetext{
* Speaker.
} 


\section{Introduction}

The emission observed from galaxies in both the local and distant Universe is dominated by star-formation and accretion processes. Both of these processes are fundamental to the formation and evolution of galaxies. Accretion sources, such as AGN, provide a major power source in the Universe and can dominate the emission in objects from distant quasars down to nearby compact stellar mass accretion sources such as X-ray Binaries. Whereas star-formation is a ubiquitous process in all galaxies that enriching the ISM of galaxies. Feedback from, and between, each of these processes is crucial to our understanding of galaxy evolution and appears to be essential if the observed galaxy luminosity function is to be reconciled with predictions from the standard hierarchical clustering models.

Radio observations, both at high and low resolution, provide one of the best diagnostics of star-formation and allow a direct view of the consequences of this process even in the most dusty effected regions toward the centre of galaxies. At arcsecond angular resolutions radio interferometers are able to trace the global radio emission from galaxies in the nearby and distant Universe. Such observations at decimetre wavelengths predominantly trace synchrotron emission which, when AGN emission is discounted, act as a superb proxy for star-formation in these galaxies [9]. However by providing high resolution $\left(\sim 00^{\prime \prime} 5\right.$ or better) the current generation of radio interferometers are also able to decompose individual local galaxies into their compact radio source populations. These populations comprises of accretion dominated source such as AGN plus the tracers of the early stages of star-formation, such as compact HII regions, superstar clusters (SSCs), as well as stellar end-points like X-ray binaries, planetary nebulae, supernovae ( $\mathrm{SNe}$ ) and their remnants (SNR). When observing the redshift Universe, sub-arcsecond resolution observations may not provide the linear resolution scales to fully decompose the galaxies but they do provide unique information regarding the sizes and structures of the radio emission from galaxies as well as critical resolved information that allows the spatial separation of the radio emission from AGN and star-formation regions.

The currently available radio arrays, along with those planned in the future such as the SKA, are able to resolves galaxies at physically important size scales ( $\sim$ few to tens of parsec at distances of a few Mpc) characterising individual sources and providing a detailed extinction-free census of the compact star-formation products across a wide range of galaxy types and the environment parameter space they inhabit. For example, surveys with sub-mJy sensitivities at $\sim \mathrm{GHz}$ frequencies, can provide a census of this type will detect all of the long-lived radio SNR within several tens of Mpc, thus providing a measure of star-formation rates within local galaxies, independent of the IR-radio correlation and obscuration corrections. Such a measure will preferentially trace high mass star-formation $\left(M>8 \mathrm{M}_{\odot}\right)$ hence providing a direct tracer of the upper part of the galaxy IMF.

\section{Star-formation tracers}

The star-formation history within individual galaxies (including the current levels of starformation) still remains a crucial physical parameter which observations are only now beginning to accurately characterise. Traditionally observations of optical line, IR and the global synchrotron emission from galaxies have often been used as proxies for star-formation. However, these tracers 
do have some fundamental flaws. In many galaxies optical emission lines are heavily obscured towards their centres, thus can require potentially large corrections. Whilst IR emission, which essentially traces the light from young stars reprocessed by dust and re-radiated at longer wavelengths, relies upon empirical interpretation of physically complex processes it to be related to star-formation. Global radio synchrotron emission also provides an alternative, and extinctionfree, indication of star-formation. However, the link between radio synchrotron emission and star-formation is also due to complex physical mechanisms (see section 2.1) which are general calibrated using the radio-to-infrared correlation $[42,5,2]$. The long-standing critical issue which remains is how to calibrate either global radio or IR emission as a measure of SF, and how this calibration varies as a function of galaxy type and environment [16].

Ultra sensitive, milliarcsecond radio images of nearby galaxies provide one method by which observations can directly address this issue in a way which is independent of complex physical emission mechanisms. Whereas low resolution radio observations of normal and star-forming galaxies trace the diffuse radio emission primarily resulting from charged particles that have escaped from old supernova remnants (SNR) [9], with adequate sensitivity, high resolution observations can be used to systematically characterise the populations of individual SF products on a galaxy by galaxy basis, critically resolving away the diffuse emission. This population census can hence be used to directly infer the levels of SF.

At high angular resolutions each individual external galaxy can be considered as a laboratory containing a large sample of discrete radio sources, which can be studied in a systematic way. When observed with high sensitivity and resolution this source population, with the exception of accretion dominated objects and in particular AGN, will consist exclusively of sources related to various key phases of the stellar evolutionary sequence. This will be a mixture of sources from the early stages of SF, such as compact HII regions, through superstar clusters (SSCs), and stellar end-points like X-ray binaries, planetary nebulae, supernovae ( $\mathrm{SNe}$ ) and their remnants (SNR).

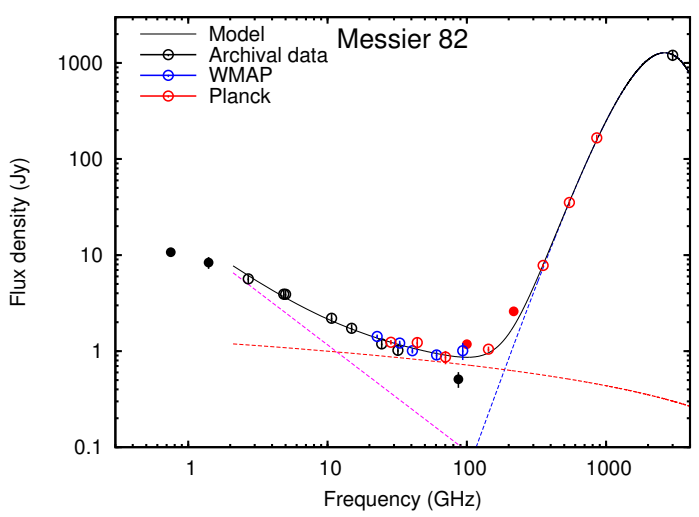

Figure 1: Spectral energy distribution of the nearby star-forming galaxy M82. Spectral fits to synctrotron (magenta), themal (free-free - red) and thermal (dust - blue) are shown alongside the combination of these products as a solid line [35].

\subsection{Radio continuum to star-formation relationship}

The large scale radio continuum emission from galaxies originates from massive stars which 
allows it to be used to trace the levels of star-formation [9]. These massive stars are the origins of the thermal radio emission. UV radiation from these stars ionizes the surrounding ISM creating HII regions which contain thermal electrons and giving rise to free-free emission (and absorption at lower frequencies). The thermal component of this radio emission is directly proportional to the level of ionising flux from the massive stars and consequently the level of current star-formation within individual galaxies.

On short stellar lifetimes these massive stars evolve into supernovae [44] which are the source of relativistic cosmic-ray electrons that are subsequently accelerate in supernova shocks. The interaction of these relativistic cosmic ray electrons with magnetic fields within the host galaxy results in the generation of non-thermal, synchrotron, radio emission. This synchrotron radio emission is the dominant diffuse radio emission from normal starforming galaxies at frequencies of $\sim \mathrm{GHz}[9]$. Provided that these cosmic ray electrons are constrained within their host galaxy, they can be considered as a pure calorimeter [41,20]. However, in the case of most galaxies some of these electrons will escape their hosts and as such more complex 'leaky calorimeter' models are required $[5,19]$. The success of these models provides the theoretical link between the non-thermal, synchrotron, radio emission and massive star-formation rates. The careful confirmation and quantisation of this link is critical since it provides an important tool in probing the levels of massive star-formation throughout the Universe.

Provided the levels of non-thermal and thermal radio emission in normal galaxies can be separated they can be used two independent extinction free star-formation tracers, with sensitivity toward current and recent massive star-formation [26]. A frequencies of $\sim \mathrm{GHz}$, where many traditional surveys have been undertaken, the emission of nearby galaxies is dominated by non-thermal emission (see Fig. 1), thus the less direct relationship of star-formation with synchrotron emission has been commonly used. However, with high sensitivity and wide frequency coverage of the radio SED of individual galaxies it is possible to separate the contribution of thermal emission ( $\sim$ few to $10 \%$ at $1->10 \mathrm{GHz}$ ), thus allowing a more direct measure of the ionising radiation from the ongoing star-formation.

\section{Views of galaxies in the local Universe}

\subsection{Surveying the products star-formations: A case study example}

The nearby starburst galaxy M82 provides a unique opportunity to study the detailed physical processes causing star-formation and the consequences of this star-formation on its surrounding environment. Within the central kpc of M82 an area of extended non-thermal and thermal radio emission traces the recent area of high star-formation activity in the galaxy. At high angular resolution (e-MERLIN resolutions) this radio emission is resolved into many tens of compact radio sources. The majority of these are discrete non-thermal sources related to individual radio supernova remnants which were originally identified in the 1980s [38, 18]. Subsequent long term monitoring and observations using a combination of VLA, MERLIN and VLBI observations by our group $[28,25,12,11,14]$ have shown the majority $(\sim 2 / 3)$ of these sources to have parsec scale shell structures, consistent with their identification as supernova remnants (SNR) with the remaining sources composing a mixture of HII regions and other source related to star-formation and accretion processes (e.g. XRB, ULX sources). 

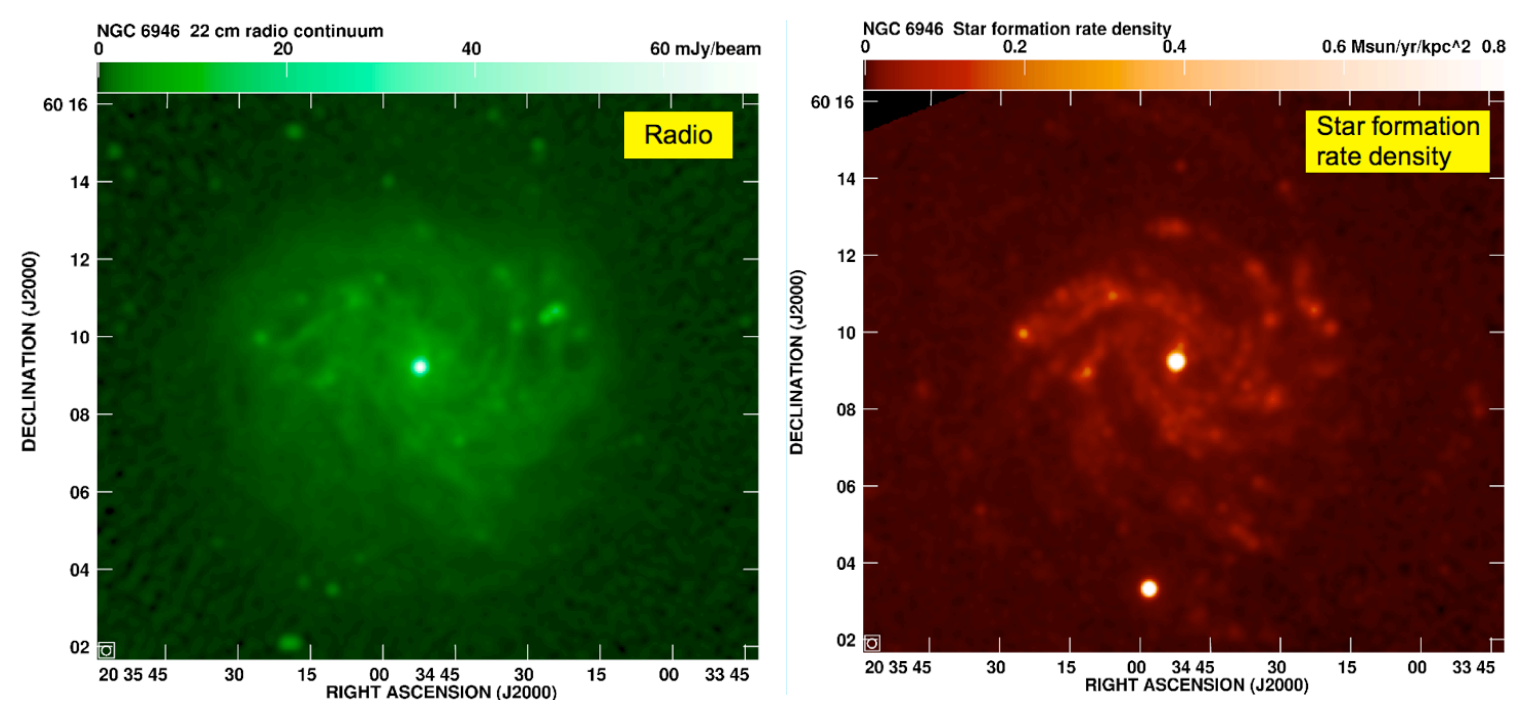

Figure 2: NGC 6946 with left: $22 \mathrm{~cm}$ radio continuum and right: SFR map

The long term radio studies of M82 have continued with e-MERLIN. With the enhanced sensitivity and image fidelity of e-MERLIN allowing a near doubling of the number of compact radio sources that can be detected and for the first time allowing the $\mu \mathrm{Jy}$ population of these sources to be unveiled, as well as a number of new and unexpected source structures. For the first time the moderately deep, high fractional bandwidth, 2014 e-MERLIN L-band and LOFAR $151 \mathrm{MHz}$ observations are constraining large scale structures within M82, including large linear shock features of sizes of a few tens of pc (see Fig. 4). The true nature of these components is yet to be fully understood, although it is likely that these are related to large scale magnetic shocks and are similar in nature and size to those found within the Galactic centre region [43]. Deep 4.5-7.5GHz e-MERLIN observations have yielded the most sensitive image of this starburst to date, imaging and resolving all of the sources in the central region with sensitivities of $10 \mu \mathrm{Jy}$. This resolution and sensitivity will allow the imaging of knot structures within all but the most compact of the RSNe. It will also provide adequate resolution to track the spatial extent of unique new sources, such as the MERLIN transient discovered in 2010 [31].

Complementing the information from high resolution centimetre wavelength interferometers such as e-MERLIN, VLA, and EVN, new instruments such as LOFAR are now providing both the sensitivity and high angular resolution to detect and image local star-forming galaxies at metre wavelengths. To obtain sub-arcsecond resolution images at frequencies of a few hundred $\mathrm{MHz}$ frequencies LOFAR utilises international baselines of $\sim 1000 \mathrm{~km}$. Several examples long baseline LOFAR observations or nearby starforming galaxies have highlighted this technique on prototypical nearby northern starburst galaxies such as M82 (see Fig 4) and Arp220 e.g. [39, 40]

\subsection{Going to the highest resolutions - resolving individual sources}

The developments of VLBI arrays over the last decade are now allowing lower surface brightness sources to be observed with milliarcsecond resolutions. However the number of sources that can be observed in local star-forming galaxies with VLBI arrays remains limited to the brightest 


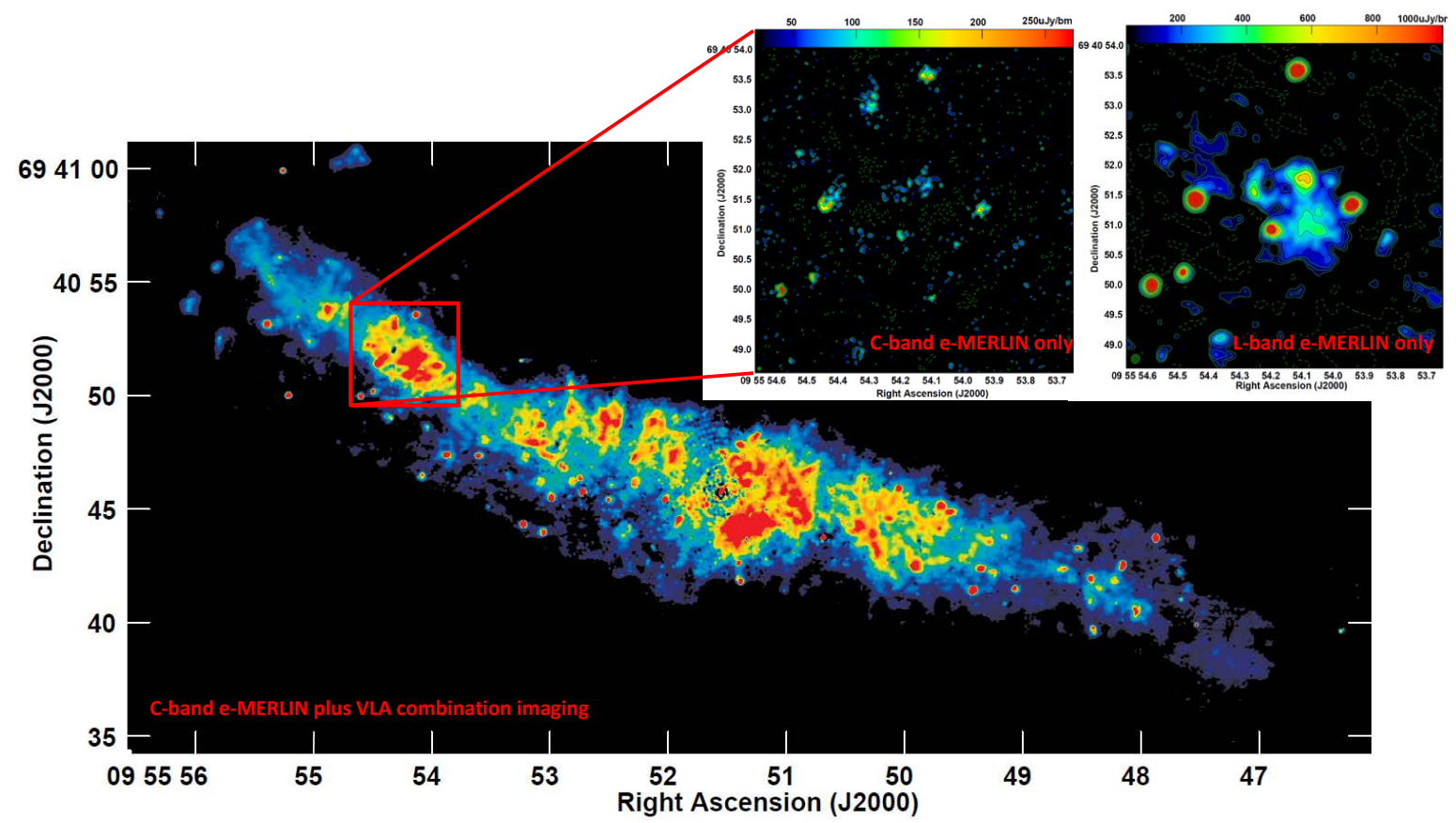

Figure 3: High resolution radio view of a small section of M82 as viewed at L- and C-band, left and right panels, with e-MERLIN, overlaid upon a combined VLA and e-MERLIN image of the central kpc of M82. Both images show a number of compact radio sources that at the 100-50mas resolution shown in these images is resolved to show descrete shells of emission from radio supernove remnants.

and most compact. In particular VLBI studies radio supernovae and supernova remnants have provided a wealth of information allowing the detailed studies of the evolution of supernovae and their interaction with the surrounding circumstellar medium. Local bright radio supernovae are relatively rare events, and to date only a handful have been intensively studied with VLBI mas-scale imaging.

One of the most well studied of these was SN1993J which exploded in M81 on 28 Mar 1993. The source was first detected at radio wavelengths at the beginning of April 1993 with intensive VLBI monitoring of the expansion and deceleration of this ongoing expansion for over 20 years see for example $[21,1,22,23]$.

More recently a new bright radio supernovae, SN2008iz, has been discovered at radio wavelengths in the centre of M82, interacting neighbour of M81 where SN1993J resided. This new source was first detected at radio wavelengths serendipitously by [6, 7] in April 2008 with the VLA, and confirmed with several other radio instruments [30, 3, 24]. Despite significant efforts at various other astronomical wavebands this source, along with many of the other more historical radio supernova remnants [11], have never been detected outside the radio part of the spectrum because of the high levels of dust extinction toward the centre of this galaxy. The gross flux density evolution of SN2008iz was comparable to that of SN993J with it reaching a peak flux density of $\sim 150$ mJy around 4 months after the supernovae explosion. Soon after the detection of this source intensive VLBI and e-MERLIN monitoring of both the structural and flux density evolution of this source was undertaken (see Fig. 5). A comprehensive study of the flux density and structural evolution of this source can be found in [17]. 

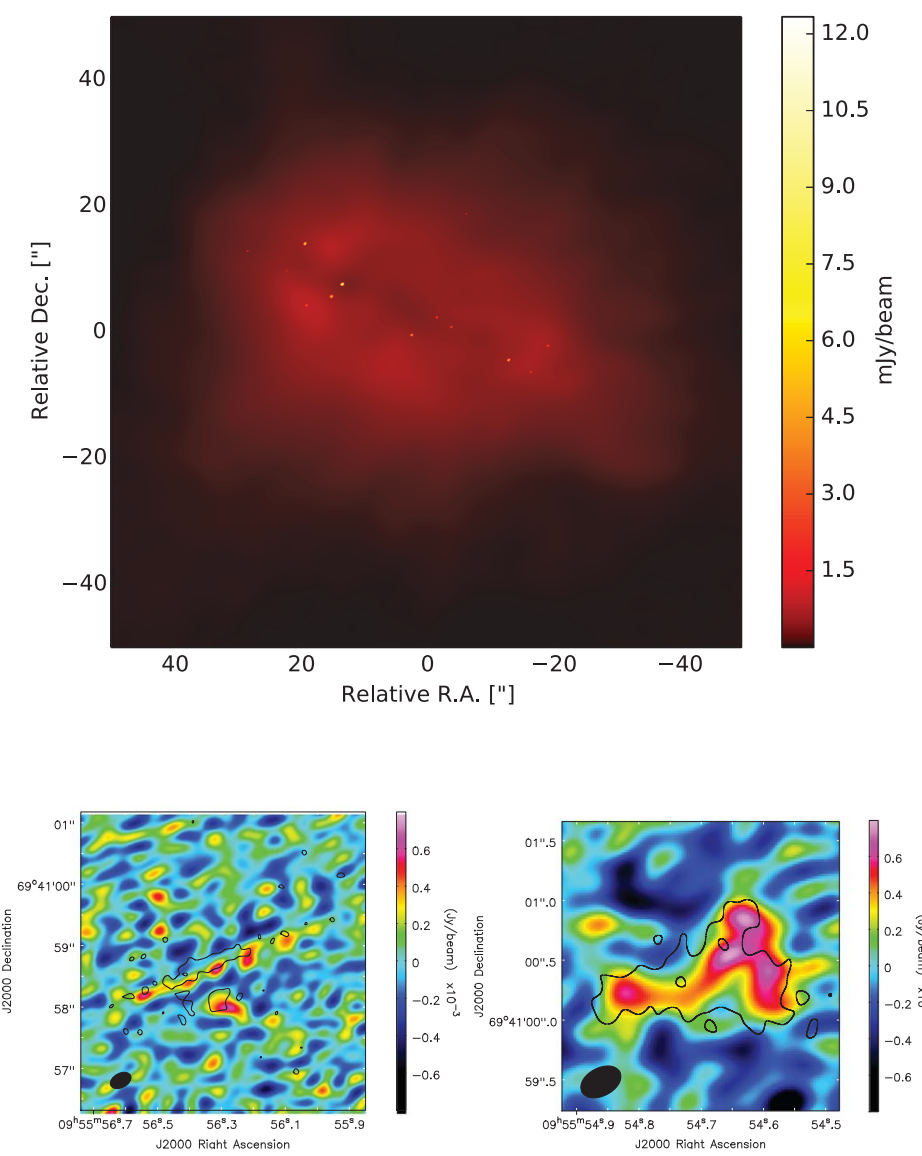

Figure 4: Top: Composite image illustrating the relative brightness between the compact and extended emission at $154 \mathrm{MHz}$ as observed with LOFAR. Bottom: Two zooms on selected regions of the M82. The bottom-left panel shows a linear feature and bottom-right shows a resolved but not clearly shell-like object. Note that the bottom-left panel covers a larger area on sky, and has lower contour levels, compared to the other three panel. Contours from e-MERLIN 1.3-1.7GHz continuum observations are ovserlaid.The LOFAR $154 \mathrm{MHz}$-beam is shown in the lower left corner. From Varenius et al (2015) [39].

\section{Extending to the high redshift}

At redshifts of 0.5 and beyond many tens of thousands of star-forming galaxies have been observed via deep field observations with the ATCA e.g. [33], VLA e.g. [10], GMRT e.g. [13], MERLIN/e-MERLIN e.g. [29, 32] and even VLBI arrays e.g. [8, 37]. These deep surveys at cmwavelengths have typically reached depths of few $\mu \mathrm{Jy}$, over areas of a few degrees or smaller, and are limited by available telescope facilities and resources. Both the depth and area coverage these will be vastly increased over the coming decade with the advent of the next generation of SKA pathfinder instruments, such as MeerKat and ASKAP, and ultimately the SKA itself [34].

The majority of the observations of extragalactic deep fields have been made using modest angular resolutions of a few arcseconds or more using instruments such as the VLA. These surveys have provided an excellent probe of the source densities of $\mu \mathrm{Jy}$ radio sources (e.g. [10]), however the vast majority of star-forming galaxies beyond the local Universe have angular sizes of around arcsecond or more, and are hence unresolved to such observations. This provides a natural limit to the information that can be derived with such observations providing little or no morphological 

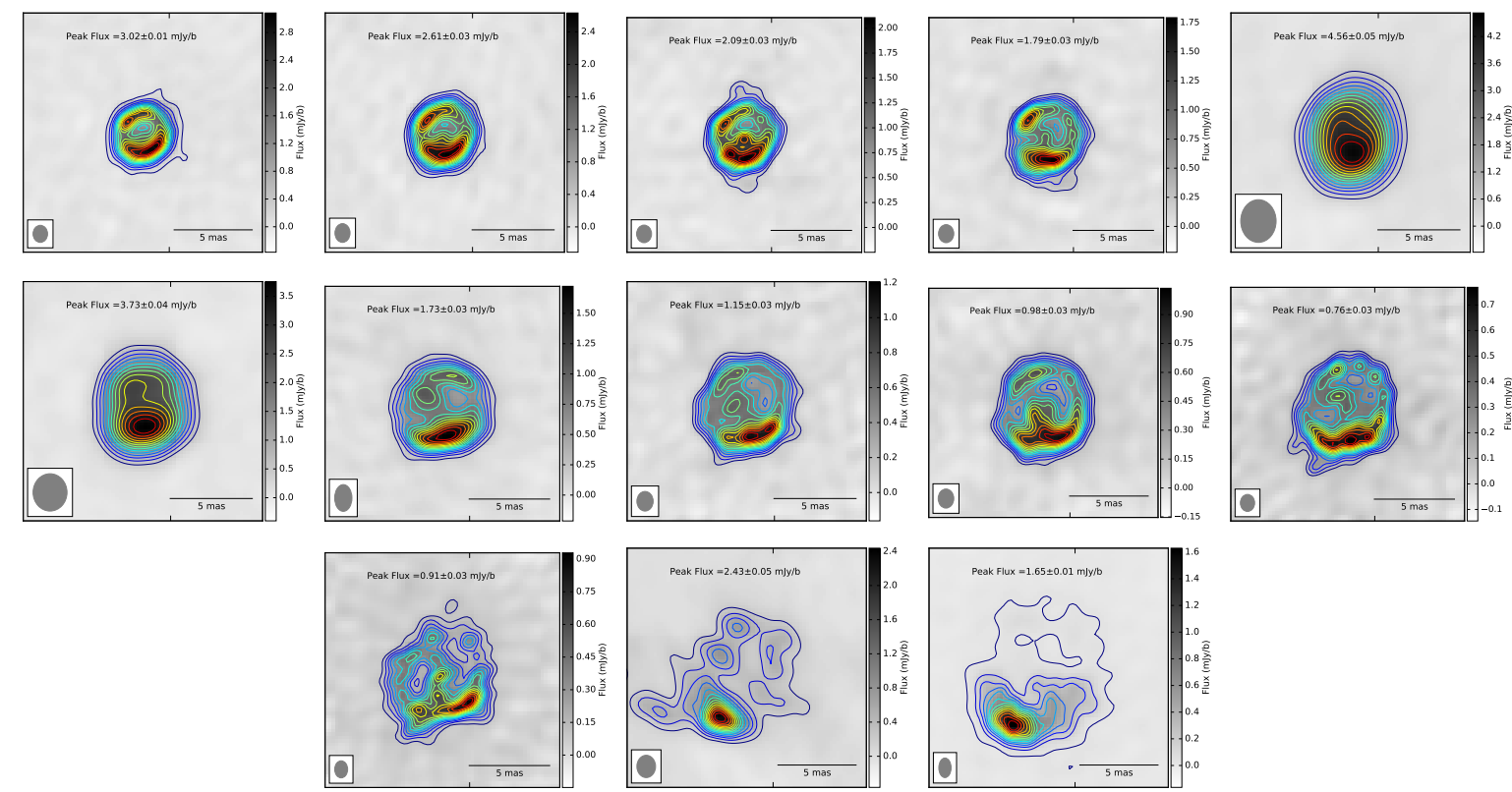

Figure 5: A series of 4.8GHz VLBI monitoring observations of SN2008iz made between days 595 and 1806 (Oct 2009-Jan 2013) following the supernovae explosion date of 18th February $2008 \pm 6$ days[24, 6]. Images from Kimani et al. [17].

information on this source population and no spatial separation via radio observations alone of the contribution of AGN emission to these galaxies. The few exceptions to this have provided deep and high resolution observations over a very limited sky area using arrays such as MERLIN and VLBI. One of the earliest and most significant of these was the work of Muxlow et al. [29] who used a combination of MERLIN and VLA data to image a few square arcminute field centred on the Hubble Deep Field to depths of $3.3 \mu \mathrm{Jy} \mathrm{bm}^{-1}$ with angular resolutions of $0 . \prime 2$ to $0 . " 4$. This project is now being superseded by the ongoing e-MERGE (e-MERLIN Galaxy Evolution) Survey [32] which is an ongoing e-MERLIN Legacy programme to observe the GOODS-N region at both $\mathrm{L}$ and C-band to sub- $\mu \mathrm{Jy}$ levels with resolutions reaching 30 mas.

One of the key objectives of e-MERGE is to track the global SFR across cosmic time. It is known that the SFR was higher in the past, but determining when this peaked occurred turns out to be a difficult task. Observing in the radio with e-MERLIN has two vital advantages: it avoids the need to estimate a dust obscuration factor, and, with an angular resolution comparable with that of the HST, it can spatially separate the different emission mechanisms in distant galaxies (Fig 6, left). e-MERGE is using these advantages in a unique way to determine the global SFR by assigning a proportion of the emission to either AGN or star forming mechanisms based upon morphological parameters. This is done statistically, rather than individually, to whole populations of galaxies. The method has been developed using the first tranche of e-MERGE data (representing about 25\%) and already hints at an increase in the SFR for galaxies at the lowest fluxes, preliminary results probing the size distribution are illustrated in Figure 6 (right) [45]. 

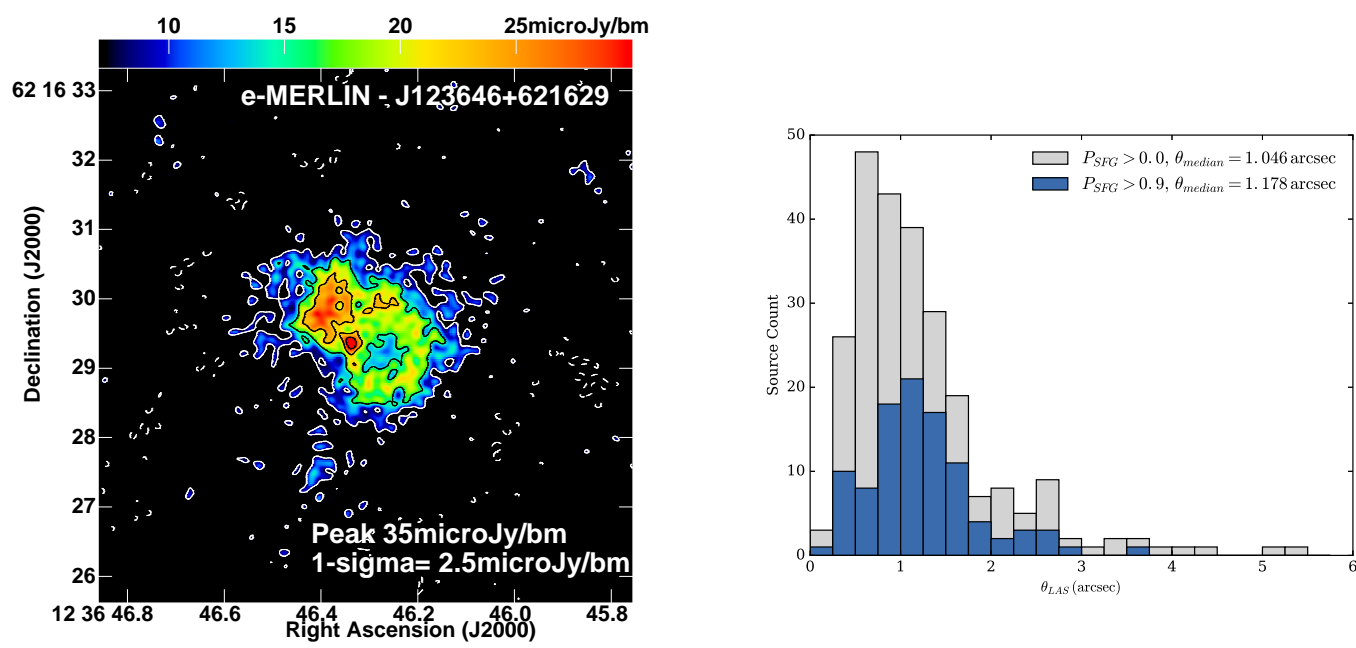

Figure 6: Left: One example starburst and AGN composite high redishift galaxy from within the GOODS-N field as observed by e-MERLIN as part of the e-MERGE project. Right: Angular size distribution of $\mu$ Jy radio sources within the GOODS-N field at $1.2-1.7 \mathrm{GHz}$ [45]

\section{Summary}

High resolution radio observations provide a unique window to study star-formation processes in the Universe. By providing resolved and spectrally separating images of the synchrotron and thermal Bremsstrahlung emission in galaxies, radio observations have can provide one of the only clean and obscured measure of the star-formation rates within galaxies. In the local Universe subarcsecond and milli-arcsecond resolution observations of the products of star-formation such as Supernovae and their remnants can be resolved both from the diffuse radio emission of galaxies, and individual sources imaged in their own right. Detailed observations of these compact radio sources with VLBI and connected element interferometers are able to resolve the temporal evolution of these source, measuring the expansion of Supernova remnants in detail and how these explosive products of star-formation interact with, and feedback energy into, the circumstellar medium of galaxies.

The knowledge now being gathered regarding the radio emission from local star-forming and normal galaxies is informing our view of the distant Universe and how star-formation effects galaxy evolution. At high redshift, deep radio surveys of otherwise blank fields are now able to image many tens of thousands of galaxies at radio wavelengths, with these radio detections providing a truly obscured measure of the star-formation history of the Universe. However, many of the radio sources at high redshifts are composite star-forming and AGN systems and the current highest resolution radio surveys are showing that these radio sources have typical angular extents of $\sim 1$ arcsecond equivalent to a few tens kpc at redshifts of $\sim 1$. As such it is becoming increasingly apparent that future deep radio surveys require sub-arcsecond angular resolutions both to characterise the sources that they detect and more importantly be able to spatially separate the contributions 
from accretion and star-formation processes in order that an unbiased census of the star-formation in the universe can be made. In this area new instruments, such as the SKA, have the opportunity to be truly transformational $[36,4,15,27]$.

\section{References}

[1] Barthel, N., Bietenholz, M. F., Rupen, M. P., Conway, J. E., Beasley, J. A., Sramek, R. A., Romney, J. D., Titus, M. A., Graham, D. A., Altunin, V. I., Jones, D. L., Rius, A. Venturi, T., Umana, G., Francis, R. L., McCall, M. L., Richer, M. G., Stevenson, C. C., Weiler, K. W., van Dyk, S. D., Panagia, N., Cannon, W. H., Popelar, J., Davis R. J., 1994, Nature, 368, 610

[2] Beswick, R. J., Muxlow, T. W. B. Thrall, H., Richards, A. M. S., \& Garrington, S. T. 2008, MNRAS, 385,1143

[3] Beswick, R. J., Muxlow, T. W. B. Pedlar, A., et al 2009, The Astronomers Telegrams, 2060, 1

[4] Beswick, R. J., Brinks, E., Pérez-Torres, M., Richards, A. M. S., Aalto, S., Alberdi, A., Argo, M. K., van Bemmel, I., Conway, J. E., Dickinson, C., Fenech, D., Gray, M. D., Klöckner, H. R., Murphy, E., Muxlow, T. W. B., Peel, M. W., Rushton, A., Schinnerer, E., 2015 "SKA studies of nearby galaxies: star-formation, accretion processes and molecular gas across all environments", in proceedings of "Advancing Astrophysics with the Square Kilometre Array", PoS (AASKA14) 070

[5] Bell, E. F. 2003, ApJ, 586, 794

[6] Brunthaler, A., Menton, K. M., Reid, M. J., Henkel, C., Bower, G. C., Falcke, H. 2009a, A\&A, 499, L17

[7] Brunthaler, A., Menton, K. M., Reid, M. J., Henkel, C., Bower, G. C., Falcke, H., 2009a, The Astronomers Telegram, 2020, 1

[8] Chi, S., Barthel, P. D., Garrett, M. A., 2013 A\&A, 550, 86

[9] Condon, J. J. 1992, ARA\&A, 30, 575

[10] Condon, J. J., Cotton, W. D., Fomalont, E. B., Kellermann, K. I., Miller, N., Perley, R. A., Scott, D., Vernstrom, T., Wall, J. V., 2012, ApJ, 758, 14

[11] Fenech, D. M., Beswick, R. J., Muxlow, T. W. B., Pedlar, A., Argo, M. K. 2010, MNRAS, 408, 607

[12] Fenech, D. M., Muxlow, T. W. B., Beswick, R. J., Pedlar, A., Argo, M. K. 2008, MNRAS, 391, 1384

[13] Garn, T., Green, D. A., Hales, S. E. G., Riley, J. M., Alexander, P., 2007 MNRAS, 376, 1251

[14] Gendre, M. A, Fenech, D. M., Beswick, R. J., Muxlow, T. W. B., Argo, M. K. 2013, MNRAS, 431, 1107

[15] Jarvis, M., Seymour, N., Afonso, J., Best, P., Beswick, R., Heywood, I., Huynh, M., Murphy, E., Prandoni, I., Schinnerer, E., Simpson, C., Vaccari, M., White, S. "The star-formation history of the Universe with the SKA", in proceedings of "Advancing Astrophysics with the Square Kilometre Array", POS (AASKA14) 068

[16] Kennicutt, R. C., Jr. 1998, ApJ, 498, 541

[17] Kimani, N., Sendlinger, K., Brunthaler, A., Menton, K. M., Martí-Vidal, I., Henkel, C., Falcke, H., Muxlow, T. B. W., Beswick, R. J., Bower, G., 2016, A\&A, submitted.

[18] Kronberg, P. P., Biermann, P., Schwab, F. R. 1984, ApJ, 291, 693 
[19] Lacki, B. C., Thompson, T. A., \& Quataert, E. 2010, ApJ, 717, 1

[20] Lisenfeld, U., Völk, H. J., \& Xu, C. 1996, A\&A, 314, 745

[21] Marcaide, J, M. Alberdi, A., Ros, E., Diamond, P., Schmidt, B., Shapiro, I. I., Baath, L., Davis, R. J., de Bruyn, A. G., Elosegui, P., Guirada, J. G., Jones, D. L., Krichbaum, T. P., Whitney, A. R., Witzel, A., Zensus, A., 1994, Nature, 372, 44

[22] Marcaide, J, M. Alberdi, A., Ros, E., Diamond, P., Shapiro, I. I., Guirada, J. G., Jones, D. L., Krichbaum, T. P., Mantovani, F., Preston, R. A., Rius, A., Schilizzi, R. T., Trigilio, C., Whitney, A. R., Witzel, A. 1995, Science, 270, 1475

[23] Marcaide, J, M. Martí-Vidal, I., Alberdi, A., Pérez-Torres, M. A., Ros, E., Diamond, P. J., Guirado, J. C., Lara, L., Shapiro, I. I., Stockdale, C. J., Weiler, K. W., Mantovani, F., Preston, R. A., Schilizzi, R. T., Sramek, R. A., Trigilio, C., van Dyk, S. D., Whitney, A. R., 2009, A\&A, 505, 927

[24] Marchili, N., Marti-Vidal, I., Brunthaler, A., Krichbaum, T. P., MÃijller, P., Liu, X., Song, H.-G., Bach, U., Beswick, R., Zensus, J. A. 2010, A\&A, 509, A47

[25] McDonald, A. R, Muxlow, T. W. B., Wills, K. A., Pedlar, A, Beswick, R. J., 2002, MNRAS, 334, 912

[26] Murphy, E. J. 2013, ApJ, 777, 58

[27] Murphy, E., Sargent, M., Beswick, R., Dickinson, C., Heywood, I., Hunt, L., Huynh, M., Jarvis, M., Karim, A., Krause, M., Prandoni, I., Seymour, N., Schinnerer, E., Tabatabaei, F., Wagg, J., 2015 "SKA Observations at >10GHz: Fundamental Astrophysics of Star-formation at High-z ", in proceedings of "Advancing Astrophysics with the Square Kilometre Array", PoS (AASKA14) 085

[28] Muxlow, T. W. B., Pedlar, A., Wilkinson, P. N., Axon, D. J., Sanders, E. M., de Bruyn, A. G. 1994, MNRAS, 266, 455

[29] Muxlow, T. W. B., Richards, A. M. S., Garrington, S. T., Wilkinson, P. N., Anderson, B., Richards, E. A., Axon, D. J., Fomalont, E. B., Kellermann, K. I., Partridge, R. B., Windhorst, R. A., 2005, MNRAS, 358, 1159

[30] Muxlow, T. W. B., Beswick, R. J., Pedlar, A., Fenech, D., Argo, M. K., Ward, M. J., Zezas, A., 2009, The Astronomers Telegram, 2073, 1

[31] Muxlow, T. W. B., Beswick, R. J., Garrington, S. T., Pedlar, A, Fenech, D. M., Argo, M. K., van Eymeren J., ward, M., Zezas, A., Brunthaler, A. 2010, MNRAS, 404, 109

[32] Muxlow, T. W. B., Smail, I., McHardy, I., et al., in proceedings of "Proceedings of the 12th European VLBI Network Symposium and Users Meeting (EVN 2014)", PoS (EVN2014) 11

[33] Norris, R. P., Afonso, J., Appleton, P. N., Boyle, B. J. Ciliegi, P., Croom, S. M., Huynh, M. T., Jackson, C. A., Koekemoer, A. M., Lonsdale, C. J., Middelberg, E., Mobasher, B., Oliver, S. J., Polletta, M., Siana, B. D., Smail, I., Voronkov, M. A., 2006 AJ, 132, 2409

[34] Norris, R. P., Afonso, J.; Bacon, D.; Beck, R., Bell, M. Beswick, R. J., Best, P., et al 2013, PASA, 30, 20

[35] Peel, M. W., Dickinson, C., Davies, R. D., Clements, D. L., Beswick, R. J. 2011, MNRAS, 416, 99

[36] Prandoni, I. \& Seymour, N. "Revealing the Physics and Evolution of Galaxies and Galaxy Clusters with SKA Continuum Surveys", in proceedings of "Advancing Astrophysics with the Square Kilometre Array", PoS (AASKA14) 067

[37] Radcliffe, J. F., Garrett, M. A., Beswick, R. J., Muxlow, T. W. B., Barthel, P. D., Deller, A. T., Middelberg, E., 2016 A\&A, 587, 85 
[38] Unger, S., Pedlar, A., Axon, D. J., Wilkinson, P. N., Appletone, P. N. 1984, MNRAS, 211, 783

[39] Varenius, E., Conway, J. E., Martí-Vidal, I, Beswick, R. J., Deller, A. T., Wucknitz, O., Jackson, N., Adebahr, B., Pérez-Torres, M. A., Chyzy, K. T., Carozzi, T. D., Moldón, J., Aalto, S., Beck, R., Best, P., Dettmar, R.-J., van Driel, W., Brunetti, G., Brüggen, M., Haverkorn, M., Heald, G., Horellou, C., Jarvis, M. J., Morabito, L. K., Miley, G. K., Röttgering, H. J. A., Toribio, M. C., White, G. J., 2015, A\&A, 574, 114

[40] Varenius, E., Conway, J. E., Martí-Vidal, I, Beswick, R. J. et al 2016, A\&A, submitted

[41] Völk, H. J. 1989, A\&A, 218, 67

[42] Yun, M. S., Reddy, N. A., \& Condon, J. J. 2001, ApJ, 554, 803

[43] Yusef-Zadeh, F., Hewitt, J. W., \& Cotton, W. 2004, ApJS, 155, 421

[44] Weiler, K. W, Panagia, N. Montes, M. J. Sramek, R. A. 2002, ARA\&A, 40, 387

[45] Wrigley, N. 2016, PhD thesis, The University of Manchester 